\title{
Narrativa publicitaria en la webradio. El esfuerzo de los anunciantes para sobrevivir
}

\author{
Aurora GARCÍA GONZÁLEZ \\ Universidad de Vigo \\ auroragg@uvigo.es
}

Recibido: 25/10/2012

Aceptado: 23/01/2013

\begin{abstract}
Resumen
El trabajo ofrece el resultado de un análisis de diferentes piezas de narrativa radiofónica sobre la base de la influencia de la web en el pensamiento contemporáneo desde la perspectiva de varios autores. El trabajo se encuadra en el ámbito de la comunicación publicitaria radiofónica con los siguientes objetivos: 1. Estudiar la evolución de la narrativa en la publicidad radiofónica desde 2000, considerado como fecha irrupción general de las nuevas tecnologías y en particular del uso de Internet. 2. Analizar qué elementos de la narración han ido cambiando como consecuencia del excesivo contagio de la red, y del nuevo modo de pensar. Y 3. Mostrar que la publicidad puede muy bien contribuir a la construcción de una sociedad sustentada en el conocimiento y los valores como formas mejores de convivencia y participación social.
\end{abstract}

Palabras clave: Radio, Publicidad, Internet, Narrativa, Relato.

\section{Advertising in the Narrative Webradio. Advertisers Effort to Survive}

\begin{abstract}
The paper includes an analysis of different radio narrative pieces based on the influence of the web in contemporary thought. The work is framed in the field of radio advertising communication with the following objectives: 1 . Studying the evolution of narrative in radio advertising since 2000, generally considered as the date of emergence of new technologies and particularly the use of Internet. 2. Analyze what elements of storytelling have changed as a result of excessive spread of the network, and the new way of thinking. And 3. Show that advertising may well contribute to building a society based on knowledge and values as better ways of living and social participation.
\end{abstract}

Keywords: Radio. Advertising, Internet, Narrative, Storytelling

\section{Referencia normalizada}

GARCÍA GONZÁLEZ, Aurora (2013): "Narrativa publicitaria en la webradio. El esfuerzo de los anunciantes para sobrevivir". Estudios sobre el mensaje periodístico. Vol. 19, Núm. especial abril, págs.: 763771. Madrid, Servicio de Publicaciones de la Universidad Complutense.

Sumario: 1 . Introducción; 1.2. Estado de la cuestión; 1.2.1. El camino hacia la interactividad; 1.2.2. El desafío de los contenidos; 1.3. Descripción del problema. 2. Metodología; 2.1. Objeto de estudio; 2.2. Hipótesis. 3. Desarrollo; 3.1. Selección de la muestra; 3.2. Resultados de la investigación; 3.2.1. Acerca del volumen de cuñas; 3.2.2. Acerca del lenguaje; 3.2.3. Acerca de la narrativa radiofónica. 4. Conclusiones. 5. Referencias bibliográficas. 6. Fuentes sonoras.

\section{Introducción}

Este trabajo presenta el resultado de un análisis de diferentes piezas de narrativa radiofónica sobre la base de la influencia de la web en el pensamiento contemporáneo a partir de autores como Lessig o Carr. El estudio se ha llevado a cabo dentro de un proyecto más amplio que analiza la producción de contenidos para radio con la intención de localizar el empleo del relato como forma preferente de distintos géneros, tanto la información, como la publicidad y el entretenimiento o la formación (audio- 
libros). El estudio detenido de estos textos permite una reflexión sobre las posibilidades que ofrece la radio en la transmisión de la cultura del significante oral.

\subsection{Estado de la cuestión}

En 1998, cuando accedí a la titularidad en el área de Periodismo, publiqué las dos vertientes que destacaban en cuanto al desarrollo de la radio: el camino hacia la interactividad y el desafío de los contenidos.

\subsubsection{El camino hacia la interactividad}

La tecnología digital ha abierto el camino hacia la radio interactiva. La técnica radiofónica ha roto la tradicional unidireccionalidad y busca hoy la puesta en marcha de nuevos servicios de comunicación interactivos. Se trata de brindar nuevas oportunidades para el medio que puedan convivir con las actuales.

La digitalización de la señal es una de las grandes transformaciones de mentalidad. La radio digital es bastante más que calidad de sonido, es la posibilidad de integrarse en el mundo multimedia con numerosos servicios complementarios y de valor añadido, esos servicios que son lo más valorado por los destinatarios según sus necesidades.

"Se ha afirmado que de ahora en adelante no podrá hablarse ya de radiodifusión sino de multimedia, entendiendo por tal la integración de todas las formas de presentar la información auditiva y visual de cualquier tipo, simultáneamente y en un marco o contexto único".(Sanabria Martín, 1994).

La tecnología digital permite que toda la información que hoy recibimos de manera dispersa llegue por un cauce único. La radio es el medio de mayor capacidad interactiva porque permite vincular de forma muy directa al oyente con el emisor. El primer paso hacia la interactividad se consiguió gracias al teléfono y sirvió para experimentar las ventajas y riesgos de la misma. Por este procedimiento, se consiguió, al menos, que los oyentes pasaran de receptores pasivos a usuarios participativos, mediante llamadas a los diversos programas para dar una opinión, entablar diálogo, participar en un debate, etc. Después, por medio de contestadores, o de otros medios, los oyentes empezaron a expresar sus intereses y las empresas a ocuparse de ofrecer los programas solicitados.

A pesar de los pronósticos contrarios, el sistema de medios todavía subsiste con los componentes que tenía al final del siglo pasado (Haye, 2011: 15). Con el advenimiento de la radio digital, se plantea la posibilidad de que la audiencia pueda participar mediante un canal de respuesta. Esto implicará que cada oyente pueda efectuar personalmente una autoprogramación según sus necesidades. Y da origen a la "radio a la carta", con programas de pago como los de la TV. Es un camino también hacia una radio personalizada, de mayor intimidad. Se vive una transformación de la comunicación grupal a la individual, que va a obligar a replantear el papel del comunicador.

Cabe señalar que el presente, en términos de interactividad, se está desarrollando de la mano del medio máximo de comunicación a corto plazo: Internet. Las emisoras que emiten su programación a través de Internet se han limitado, por el momento, a convertir el sonido radiofónico en informático. Un programa de radio convencional 
con varias horas de duración, tal como se ha venido haciendo hasta ahora, es poco probable que consiga una audiencia estable ya que, para que el usuario pueda escuchar en tiempo real programas con muchos fundidos de voz y música, éstos son digitalizados en baja calidad y difundidos a través de la línea telefónica, lo que produce un sonido a veces ininteligible, y con frecuencia la saturación de la red.

Aún no se han encaminado los esfuerzos a mejorar la programación sino la tecnología. La radio detuvo la oferta de contenidos y su actitud cultural sustituyéndola por la oferta tecnológica y con ello cedió parte de su carácter mediático para volver a su función instrumental de los primeros años.

\subsubsection{El desafío de los contenidos}

La revolución tecnológica tiene un segundo efecto que es multiplicar las oportunidades para la radio. Se ha señalado que "La radio digital hertziana, por cable o por satélite, supone un incremento exponencial del número de emisoras, programaciones o servicios a disposición de los oyentes". (Martí, 1991)

El problema es de creatividad. La radio digital afronta, como vemos, el problema de los contenidos.

"La concepción actual de la radio puede resultar viciada por los condicionamientos instrumentales, mercantiles y políticos que le han impuesto una actividad frenética junto a una ausencia de reflexión. Nace así un inmovilismo teórico y práctico que congela la expresión y entroniza los instrumentos. La causa última de esta situación es una crisis de ideación. Si la ideación falla, no hay proceso radiofónico, sea o no expresivo, haga o no, referencia al contenido" (Faus, 1995: 14).

Más allá del debate sobre los procedimientos, los plazos y los aspectos jurídicos, lo que se ve necesario definir son los contenidos y servicios de este nuevo sistema. La programación es la llave que puede hacer despegar definitivamente la nueva radio.

La radio digital requiere programas novedosos e interesantes. Que se incentive la creatividad para afrontar la competencia de los nuevos soportes. Las emisoras son conscientes de que sus programaciones ya no son creadoras sino detectoras de tendencias sociales. De que ya no son ellas las que marcan las tendencias sino que intentan detectarlas y servirlas.

La radio digital puede traer consigo la segmentación de las audiencias y la especialización de las ofertas. Como señalan diversos autores “[...] están aportando estructuras empresariales con mayores posibilidades para negociar la publicidad local y para producir contenidos más atractivos" (Román Portas, 2005: 237). También es creación de radioconsumo al haber cambiado la actitud del oyente, de su pasividad ante algo que le llega y su disposición a buscar algo concreto en el mercado. Se gesta un cambio profundo en la sociedad y consecuentemente en la audiencia. Una audiencia joven está imponiendo la exigencia de una mejor calidad global, no sólo de sonido sino de contenidos. En el universo de la radio, estos cambios generan lo que Gustavo Cardoso (2009) llama edad de la radio. Y las emisoras deben constituirse en proveedoras de nuevos contenidos y dejar la gestión de la red a los operadores. Junto a las cadenas de radiodifusión se configuran ahora los operadores de red y los proveedores de 
programas. Esto va a requerir una mejor formación de los profesionales que hará más seria la industria radiofónica. A las emisiones de audio se añaden los servicios asociados y valores añadidos. Los oyentes dejan de ser sólo oyentes para convertirse en usuarios de una oferta multimedia. A un medio real se suma un medio virtual.

\subsection{Descripción del problema}

Diez años después de publicar las anteriores reflexiones, en 2009, en Bruselas y en el seno de la ECREA se puso de relieve la siguiente cuestión: el universo de la narrativa radiofónica se encuentra en pleno desarrollo, y parece incluso estar viviendo un nuevo nacimiento. Poco después, en 2011, Nicholas Carr publicaba su obra Superficiales, un libro que desarrolla la premisa ¿qué está haciendo Internet con nuestras mentes? Ambos permiten afirmar que nada de lo señalado en 1998 ha desaparecido. Las dos vertientes continúan siendo perfectamente válidas. Investigaciones publicadas en 2012 vuelven a poner el acento en la necesidad de atender a los contenidos. En términos de Emma Rodero:

"[...] de nuevo apunta a la escasa efectividad general de este tipo de publicidad radiofónica como, por otro lado, coinciden en afirmar varios autores desde hace tiempo, lo cual constituye una importante llamada de atención sobre el tipo de publicidad radiofónica que se continua haciendo en nuestro país." (Rodero, 2011: 256).

Y como sostiene Denis Renó: "es posible desarrollar una forma de producir contenido para los medios hipermediáticos y para la sociedad contemporánea". (Porto, 2008). Este punto de partida establece la opción científica que dio origen a nuestro proyecto.

\section{Metodología}

La investigación que se planteó propone el análisis de diferentes piezas de narrativa radiofónica sobre la base de la influencia de la web en el pensamiento contemporáneo siguiendo a autores como Lessig (2008) y Carr (2011). Para llevarlo a cabo se identificaron diferentes ámbitos de estudio dentro de la producción de contenidos para radio.

En particular se trata de localizar el empleo del relato como forma preferente en distintos géneros radiofónicos, tanto los informativos, como la publicidad o el entretenimiento y la formación (audiolibros).

\subsection{Objeto de estudio}

Hasta el presente la investigación que se ha llevado a cabo se centra en el análisis de la publicidad radiofónica. Como se trata de una investigación en curso, de momento, no se ha realizado la comparación entre los diferentes géneros. Pero los obtenidos en el ámbito de la publicidad son ya de por si suficientemente expresivos. Los primeros pasos de este trabajo han estudiado la narrativa publicitaria radiofónica a través de diferentes cuñas procedentes del período comprendido entre 2000 y 2010. Los objetivos que se planteaban fueron:

1. Estudiar la evolución de la narrativa en la publicidad radiofónica a partir del 2000 , considerado de interés general por la irrupción de las nuevas tecnologías y en particular por la generalización del uso de Internet. 
2. Analizar qué elementos de la narración han ido cambiando como consecuencia del excesivo contagio de la red, y del nuevo modo de pensar.

3. Y mostrar que la publicidad puede muy bien contribuir a la construcción de una sociedad sustentada en el conocimiento y los valores como formas mejores de convivencia y participación social.

\subsection{Hipótesis}

La hipótesis de partida fue que los cambios que Internet ha introducido en el modo de pensar contemporáneo, han provocado cambios también en la narrativa radiofónica. Y es conveniente profundizar en su conocimiento como recurso para optimizar la eficacia comunicativa, y por ende la comunicación publicitaria.

\section{Desarrollo}

Para este análisis, se seleccionaron los textos de las cuñas que formaron parte de distintas campañas presentadas al Festival de San Sebastián, llamado posteriormente El Sol, entre los años 2000 y 2010, como se ha dicho. Se trata de un corpus de trabajo integrado por más de 500 anuncios considerados como los mejor construidos, los más expresivos y no preparados ex profeso para la investigación.

Para verificar la hipótesis de trabajo se procedió, por medio de grupos de escucha, a la identificación de constantes en los textos de estas cuñas, referidas tanto a la forma como al contenido y la duración de las mismas. Se ha utilizado como referencia bibliografía de autores como Nicholas Carr, Lawrence Lessig y otros. El análisis detenido de las cuñas permite establecer el alcance del objetivo tres, y posteriormente complementarlo con aportaciones en torno a los resultados por medio de grupos de discusión.

Para establecer un estudio comparativo que pudiera arrojar nuevas luces sobre la investigación se procedió a elaborar una ficha metodológica detallada. Esta ficha se aplicó en un análisis cronseccional del primer año en que participaron en el Festival cuñas españolas e iberoamericanas, con sustanciales diferencias. Por el momento se ha emprendido el análisis comparativo entre cuñas procedentes de ámbitos culturales muy distintos pero con una lengua común. Está previsto el análisis comparativo que incluya también cuñas en lengua portuguesa, para lo que se realizará una nueva selección cronseccional de una de las últimas ediciones.

\subsection{Selección de la muestra}

Inicialmente se planteó estudiar la totalidad de las cuñas presentadas a los festivales de publicidad celebrados en San Sebastián desde el año 2000. Dadas las características del certamen y la evolución que vivió, fue necesario realizar algunas acotaciones.

Este festival había incorporado por primera vez la categoría "radio" en 1996. Desde entonces y en sucesivas ediciones se fueron añadiendo nuevas categorías: publicidad interactiva, marketing directo, marketing promocional. Pero el verdadero cambio del certamen se produjo en la décimo séptima edición (2002) cuando el festival se abrió a Portugal, América Latina y a los Estados Unidos hispanos (EUA). En 2006 el medio radio duplicó su participación. Este incremento que se afianzó en 2007 estuvo causado 
por la apertura del festival a Latinoamérica, donde la publicidad radiofónica está mejor considerada, y por la disminución de la cuota de inscripción del festival. Por eso, en este trabajo, a partir de 2005, se han utilizado únicamente los anuncios que obtuvieron algún premio en las respectivas ediciones del festival.

Finalmente se analizaron 508 cuñas distribuidas de la siguiente manera: año 2000 (42); año 2001 (154); año 2002 (91); año 2003 (114); año 2004 (47), y los anuncios que fueron premiados entre los años 2005 y 2010 (60).

\subsection{Resultados de la investigación}

Para este artículo se ha organizado y resumido el resultado del trabajo en torno a tres aspectos en los que es posible advertir con mayor claridad cambios: el volumen de cuñas, el lenguaje y la narrativa radiofónica. Se trata de tres escenarios de la investigación en lo que se ha trabajado y que permiten una ordenación clarificadora del material.

\subsubsection{Acerca del volumen de cuñas}

Con respecto al primer objetivo de este trabajo que era estudiar la evolución de la narrativa en estos años tras la irrupción de Internet, se percibe una interacción gradual y progresiva. Lo primero que se advierte en el volumen de anuncios que constituyeron el universo de estudio es la presencia creciente en los primeros años de cuñas de carácter informativo destinadas a dar a conocer servicios punto com o portales web. Se trata de marcas relacionadas con la comunicación: telefonía móvil y portales y servicios de internet, como "Yupi", "idealistas.com" o "TPI-páginas amarillas on line".

En el arco de tiempo estudiado destacan dos años: el año 2001 fue en términos absolutos el año en que se encontró una mayor cantidad de anuncios que aluden a la web de un modo u otro. Posiblemente esto obedezca a la universalización de Internet, a partir del año 2000; coincidiendo con este crecimiento del uso de Internet y su difusión a todos los ámbitos de la vida se registra igualmente un mayor número de anuncios cuyos contenidos incluyen alguna referencia al reciente medio.

Por otra parte resulta también significativa la fecha de 2005 en que parece que la tendencia da un vuelco y se constata que a partir de ese año ya las cuñas no hacen referencias explícitas a la novedad de la red sino que ésta, con sus posibilidades y su nuevo lenguaje se encuentra incorporada de modo sutil, no explícito. Esto se ve mejor en el uso del lenguaje publicitario.

\subsubsection{Acerca del lenguaje}

La constatación del descenso del número de cuñas que hablan directamente de Internet evidencia la integración de la red en la cotidianidad. Todo el lenguaje en el que se redactan las cuñas se encuentra permeabilizado con términos web. Ya no se trata de contenidos informativos, más bien explicativos de la novedad, sino que el habla y la lengua resultan afectadas por esta proximidad. Y esto no sólo en los términos que se utilizan sino hasta en la forma de pensar o de encarar los asuntos tal como se reproduce en esos diálogos.

Entre los cambios que ha introducido Internet uno de los más importantes ha sido modificar el modo que tienen las personas de acceder al conocimiento. Siguiendo a 
Carr (2011), como él a McLuhan (1962), hay que admitir que al final de la década estudiada nos encontramos con una audiencia que dispone de un elevado volumen de información, pero con mucha menos capacidad de concentración y menos resolución a la hora de tomar decisiones; principalmente si se trata de encarar y resolver problemas. Algunas de las cuñas estudiadas lo ponen de manifiesto.

La búsqueda de información y de soluciones, algo imprescindible en la comunicación publicitaria, se ha hecho más fácil y más rápida gracias a los buscadores y a la implementación de las redes. Y sin embargo, pese a los avances de la ciencia que se centran en intentar llegar al algoritmo perfecto en cuanto a la inmediatez de la búsqueda, la memorización se torna cada vez más difícil (Rodero, 2012). Las personas tienen inexorablemente menor capacidad de retener datos y se pierde el hábito del esfuerzo como consecuencia de la facilidad con la que se cuenta para localizar lo que se busca.

\subsubsection{Acerca de la narrativa radiofónica}

Otro objetivo prioritario de este trabajo era analizar concretamente qué elementos de la narrativa radiofónica, se han visto modificados como consecuencia del contagio de la red. En este sentido cabe sostener que: las cuñas analizadas muestran que sigue siendo habitual el empleo de la narrativa tradicional, tal como siempre se ha hecho en la publicidad de la radio. Y de la misma manera se constata que el valor de la palabra sigue siendo prioritario en la radio para la comunicación de ideas. En este sentido subraya Menesses (2012) que la radio de palabra, informativa o no, tiene más posibilidades de sobrevivir que la radio musical.

La palabra radiofónica recupera su protagonismo en la narrativa publicitaria contemporánea como expresión del pensamiento pero acusa también cierta pobreza del mismo y pone de relieve el pensamiento débil tan característico de las sociedades posmodernas.

No es interesante transcribir en estas páginas los anuncios estudiados. Pero en los textos estudiados se observa que son cada vez más breves, con un máximo de 15 segundos ya que se considera que todo lo que excede ese límite es tiempo histórico (Nieto Tamargo, 1991). Las cuñas estudiadas hacen un uso pleonástico de palabras banales, apoyadas en el diálogo interactivo, y reflejan historias de la vida cotidiana. Esta vida cotidiana a su vez transparenta unas relaciones cada vez más virtuales y unos entornos de trabajo también virtuales.

El resultado refleja una sociedad que en lugar de ser del conocimiento es una sociedad desconcertada (Muñoz Zayas 2011: 163), incapaz de generar pensamiento. Y esto contribuye en buena medida a transmitir una imagen reductiva de la persona que a fin de cuentas es el destinatario de los mensajes publicitarios.

\section{Conclusiones}

El estudio llevado a cabo permite establecer, bien que provisionalmente, al menos las siguientes conclusiones:

- Para la radio no es nueva la situación de escucha en segundo nivel, incluso en un tercer y cuarto plano. Por eso en contra de lo que nuestra hipótesis proponía, la ela- 
boración de mensajes publicitarios radiofónicos no se ha visto afectada por el advenimiento de la red, tanto como lo han sido otros medios.

- Pero sí se comprueba la creciente falta de atención en la audiencia, incluso la incapacidad para prestar atención de manera continuada durante los 15 segundos que suele durar una cuña; los anunciantes y principalmente los creativos publicitarios tienen que emprender una lucha mucho más intensa por retener una atención cada vez más solicitada por diferentes impactos.

- Este esfuerzo se ha percibido de entrada en el empleo del relato como forma preferente o muy habitual en la publicidad radiofónica.

- En esta situación es posible afirmar que la competencia no la constituyen otros medios de comunicación, ni otros anunciantes, ni siquiera Internet, sino que la competencia contra la que habría que luchar es la dependencia tan fuerte que el oyente tiene de las nuevas tecnologías.

- Para la publicidad, el nuevo entorno multitasking constituye un desafío aún mayor debido no sólo a la división de la atención de los oyentes sino incluso a la necesidad imperiosa que este entorno genera de hacer inmediatamente, otra cosa. Todo lo que no sea estar conectado, no retiene su pensamiento, ni le retiene a él.

- El impacto de Internet en el discurso publicitario está someramente planteado y está claro que hace falta una mayor reflexión desde el ámbito académico y científico para proponer soluciones a los problemas que plantea.

\section{Referencias bibliográficas}

CARR, Nicholas (2011): Superficiales. Barcelona, Taurus.

FAUS, Ángel (1995): La era audiovisual. Historia de los primeros cien años de la radio y la televisión. Barcelona, Ediciones Internacionales Universitarias.

HAYE, Ricardo (2011): "Sobre o rádio do futuro" en Rádio-Leituras. Año II, Num 01 Edición enero-julio. Universidad Federal de Santa María (Brasil) ISSN: 21796033. Disponible en http://radioleituras.wordpress.com

LESSIG, Lawrence (2008): Remix. London, Bloomsbury Academic.

MARTÍ MARTÍ, José (1991): Modelos de programación radiofónica. Barcelona, Feed-back.

MARTÍ MARTÍ, José (1996): "Los profesionales ante el reto tecnológico: creatividad e influencia social" Fundesco $\mathrm{N}^{\mathrm{o}} 182$. Noviembre, p. 16.

MCLUHAN, Marshall (1962): Understanding media. The extensions of man. Berkeley (CA), Gingko Press.

MENESES, Joao Paulo (2012): Estudos sobre a rádio. Passado, presente e futuro. Porto, Mais Leituras.

MUÑOZ ZAYAS, Rafael (2011): “"¿Qué está haciendo Internet con nuestras mentes? Superficiales». Nicholas Carr." En Extoikos, n 2, p. 163

NIETO TAMARGO, Alfonso (1990): “Marketing e Información”, en Comunicación y Sociedad, vol. III, núms. 1 y 2, p. 81. 
PORTO RENÓ, Denis (2008): "El montaje audiovisual como base narrativa para el cine documental interactivo: nuevos estudios", en Revista Latina de Comunicación Social, 63, pp. 83-90, en pdf. La Laguna (Tenerife), Universidad de La Laguna. Recuperado el 29 de julio de 2012 de: http://www.ull.es/publicaciones/latina /_2008/08_Brasil/2008_Denis_Porto.html.

RODERO ANTÓN, Emma (2011): "Posición serial, densidad informativa y velocidad de lectura en el recuerdo de las cuñas de radio" en Pensar la Publicidad, vol. 5, $\mathrm{n}^{\circ}$ 2, 255-276

ROMÁN PORTAS, Mercedes (2005): "La televisión local en España" en Sphera Publica $\mathrm{n}^{\circ}$ 5. Murcia, Servicio de Publicaciones de la UCAM.

ROMÁN PORTAS, Mercedes (2008):“Televisión digital terrestre local en Galicia" en Ámbitos. Revista Internacional de Comunicación, $\mathrm{n}^{\circ}$ 17. Sevilla, Departamento de Periodismo II.

SANABRIA MARTÍN, Francisco (1994): Información audiovisual: Teoría y técnica de la información radiofónica y televisiva. Barcelona, Bosch.

VIEIRA, José. CARDOSO, Gustavo. y MENDONÇA, Santiago (2012): "Os novos caminhos da rádio: Radiomorphosis. Tendências e Prospectivas" en Obercom. Porto.

\section{Fuentes Sonoras}

- Cuñas radiofónicas presentadas a los Festivales de Publicidad de San Sebastian 2000 a 2005.

- Archivo histórico de E1 SOL. http://www.elsolfestival.net/ (14/03/2012)

\section{Aurora GARCÍA GONZÁLEZ}

Universidad de Vigo

Facultad de Ciencias Sociales y de la Comunicación

Profesora titular de Periodismo.

auroragg@uvigo.es 